nothing when the ion is in the $S$-state, (for example, $\mathrm{Gd}+++)$ is significant. Indeed, from the known magnetic anisotropy of the group in the crystal state, and its birefringence, a rough estimate can be made of the magnetic double refraction of the solution, and it is found to be of the right order of magnitude.

Indian Association for the

K. S. KRISHNAN.

Cultivation of Science, Calcutta.

Feb. 15.

\footnotetext{
${ }^{1}$ Proc. Roy. Soe., A, 161, 220 (1937) ; 164, 117 (1938).

${ }^{2} J$. Chem. Phys., 6, 297 and 654 (1938).

${ }^{3}$ Raman, C. V., and Chinchalkar, S. W., NATURE, 123, 758 (1931); Haenny, C., C.R., 193, 931 (1931); 195, 219 (1932). ' Phil. Mag., 20, 856 (1935).
}

\section{A Comprehensive Fundamental Electrical Formula}

Dr. C. V. Drysdale has pointed out that his formula 1 can be applied to the calculation of what are usually called 'electromagnetic forces'; and it then becomes an expression (in vector notation) for the generally accepted principle called the BiotSavart law. But the experiments to which I referred in NaTure of August 7, 1937, have shown that the Biot-Savart law sometimes indicates only a small fraction of the actual force, and have therefore shown the serious incorrectness of the more general formula proposed by Dr. Drysdale.

The accuracy of my experimental results was verified by finding that they were all in close agreement with the formula given in Maxwell's Art. 583. When applied to the case shown in Fig. 2 of my above-mentioned communication to NATURE, this formula becomes

$$
F=\frac{1}{2} i^{2} d L / d y,
$$

where $F$ is the force that acts in the vertically downward direction on $M B C N$, while this part of the circuit moves through the distance $d y$ in that direction, which movement increases the inductance by the amount $d L$. The value of $d L / d y$ can be found by differentiating the well-known expression for the inductance of a rectangular circuit. Maxwell's formula says that the vertical force exerted on $M B C N$ by $M A D N$ and on $M A D N$ by $M B C N$, is $8 \cdot 74 i^{2}$, whereas Drysdale's formula says that the force exerted on $M B C N$ by $M A D N$ is $0.43 i^{2}$, and that the force exerted on $M A D N$ by $M B C N$ is $0 \cdot 17 i^{2}$, this last being less than one-fiftieth of the actual force. Here it will be seen that Drysdale's formula contradicts, to a very serious extent, both Maxwell's formula and Newton's laws of motion.

46 Grosvenor Road, W. F. Dunton.

Birmingham, 20. Jan. 30

${ }^{2}$ NATURE, 142, 995 (1938).

Mr. DunTon's measurements do not in any way affect the validity either of my equation, the BiotSavart law, Faraday's law of electromagnetic induction, or the magnetic flux-cutting equation. His error lies in assuming that it is only the magnetic field of the lower fixed portion $M_{1} A D M_{2}$ of the accompanying circuit which produces a force on the conductor $B C$; whereas the only part of the magnetic field produced by the whole circuit which does not produce a force on $B C$ is that due to $B C$ itself. If for simplicity we consider a very long narrow rectangular loop of breadth $b$ and conductor radius $a$, the magnetic

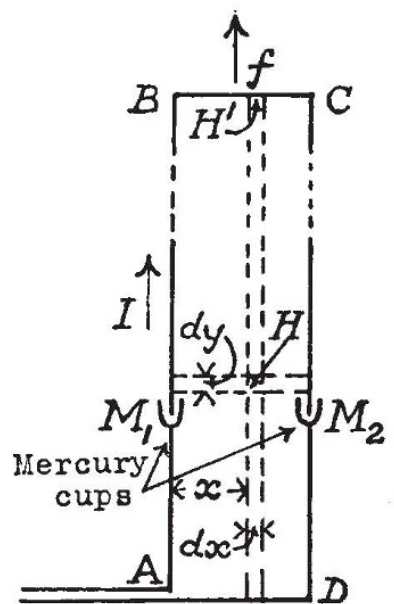

force at any distance $x$ from $A B$ and remote from $B C$ or $A D$ is $H=2 I / x+2 I /(b-x)$, and hence if the length of the loop is increased by the amount $d y$

$$
\frac{\partial I}{\partial y}=2 \int_{a}^{b}\{1 / x+1 /(b-x)\} d x
$$

neglecting the internal magnetic field in the conductors. But the magnetic force at a corresponding point on $B C$ is $H^{\prime}=H / 2$, and the force on $B C$ or on $A D$ is therefore

$f=I \int_{a}^{b} H^{\prime} d x=I^{2} \int_{a}^{b}\{1 / x+1 /(b-x)\} d x=\frac{1}{2} I^{2} \frac{\partial L}{\partial y}$,

which is Maxwell's formula, with which Mr. Dunton's measurements agreed. He ought actually to have observed a somewhat greater force due to the pressure on the ends of the conductors caused by the constricting effect of the current in the mercury (the 'pinch phenomenon' of Carl Hering), which is covered by the same argument; but the above is sufficient to show that his supposed discrepancy has no foundation.

\section{V. Drysd ale.}

\section{Frequency Interpretations in Probability}

If he were consistent, de Moivre would not have accepted 5/12 as the probability in Dr. Jeffrey's problem. (Two boxes, one containing 1 white and 2 black balls, the other 1 white and 1 black. Choose a box; then draw a ball; what is the probability of drawing white? Nature, Feb. 11.) For, according to his definition, the statement that the probability is $5 / 12$ must mean that there are $12 n$ different possible cases, of which $5 n$ are favourable, $n$ being an integer. That simply is not true.

The 'modern' who affirms 5/12 must hold that probability means something other than the ratio of favourable to possible cases. When the cases are sums of "equally possible" elements, then the probability happens to be that ratio, but not otherwise. De Moivre's definition, or Laplace's modification of it, is not wrong; it is merely unduly limited; it tolls us nothing about probability except in a limited class of problems. 